this means we improved the end-results in seven patients. The others refused the secondary operation and adjusted themselves to their work.

In the repair of tendons beneath the carpal ligament we usually use free grafts. If the median nerve is divided we suture it at the same time. In late cases with multiple damage to the tendons in this area we have sometimes been obliged to excise

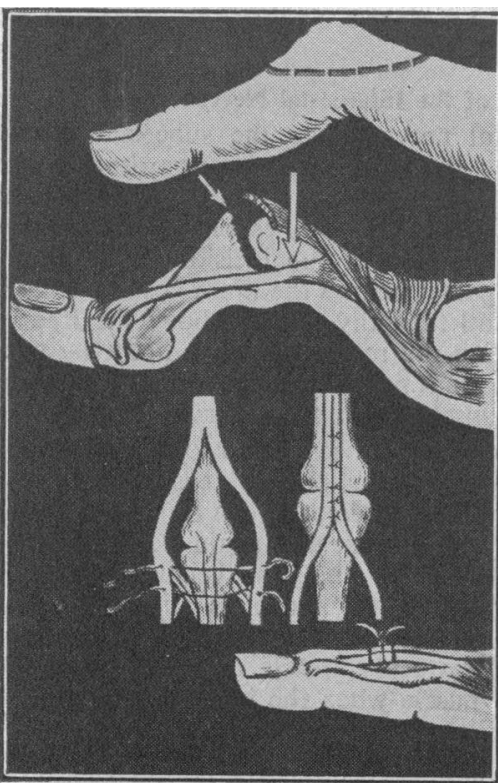

Fig. 2.-Pathology and treatment of injury to the extensor of the proximal interphalangeal joint. the carpal ligament in order to envelop the tendon suture with loose cellular tissue.

Considerable attention has been paid by us to injuries of the extensors of the proxima 1 interphalangeal joints. The patient loses the capacity to extend the middle phalanx and the shortened muscles provoke hyperextension of the distal phalanx. We have attempted to repair this by a method worked out by Weinstein (Fig. 2)

A long curved incision reaching to the middle of the base of the phalanx exposes the extensor aponeurosis over the proximal joint. The proximal aponeurosis is dissected out so as to define the lateral bundles. These are approximated with the finger in extension, with five or six fine sutures. The proximal stitches include not only the lateral bundles but also the middle bundle of the long extensor (Fig. 2).

\section{Post-operative Treatment}

After tendon repairs it is necessary to immobilize the hand in plaster for four weeks, to prevent retraction of the sutured tendon ends. Following repair of the flexor tendons, the plaster is applied to the back of the hand and forearm to stabilize the fingers in a position of flexion. In injuries of the extensor tendons the splint is applied to the palmar surface with the fingers extended.

The hand is elevated for three to four days after operation. Stitches are removed on the 9 th or 10 th day, and the patient is discharged from hospital. From the 21st day movement of the fingers is allowed. After six to eight weeks the patient returns to light work, progressing gently to full work after three months.

Aftercare includes physiotherapy and baths, started from the 15 th day. The patient also receives corticotrophin, which we have found to help in preventing scar formation and in the absorption of scarring around the repaired tendons. The surgeon who has carried out the operation must keep his patient under personal observation, and should certainly see him at least twice a week. At the end of the third week the surgeon must direct active exercises, with gentle use, to ensure the best possible function.

\section{Conclusion}

In extensor tendon injuries, plastic repairs have given satisfactory 'results in $85 \%$ of the patients. Tendon repair on the palmar surface has given satisfactory results in $77 \%$, including $65 \%$ in the area of "no-man's-land."

\section{INCISION FOR GALLBLADDER SURGERY}

BY

\author{
D. C. CORRY, M.D., F.R.C.S. \\ Surgeon, the United Oxford Hospitals
}

The two incisions generally used for operating on the gall-bladder are the right upper paramedian and Kocher's incision. The paramedian incision is often chosen when the costal angle is narrow, and Kocher's when the costal angle is broad.

Kocher (1895) described his incision as an obliquely transverse incision 10 to $15 \mathrm{~cm}$. long, made at a distance of 4 to $6 \mathrm{~cm}$. from the costal margin. As generally carried out in this country it is 2 to $3 \mathrm{~cm}$. from and parallel to the costal margin, care being taken at its lateral extremity to spare the ninth costal nerve. I was deterred from using Kocher's incision by seeing post-operative scar herniae after its employment, but for the past seven years have utilized with increasing frequency a modification which has the advantage of dividing the rectus sheath in the direction of the fibres of its main component-namely, the aponeurosis of the internal oblique. As a result, any straining after operation by tensing the external and internal oblique and transversalis abdominal muscles tends to approximate the fibres of the rectus sheath. It is essentially a muscle-splitting operation so far as the rectus sheath is concerned and so is easy to close, and the stitches do not subsequently cut out. Compared with any vertical incision the patient is far more comfortable after operation, breathing, bending, and moving easier. Normally, patients get out of bed the day after operation. I have never seen a scar hernia follow its use.

\section{Technique}

The incision (Fig. 1) is from the midline, one handbreadth $-3 \frac{1}{2}$ in. $(9 \mathrm{~cm}$.)-above the umbilicus to the tip of the tenth right costal cartilage. In some fat patients there is a natural skin crease in this line which can be utilized. The skin,

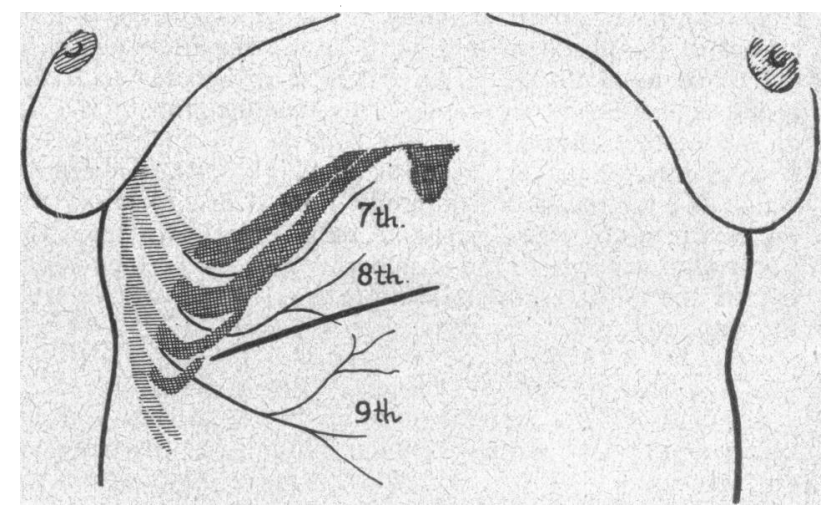

Fig. 1.-The line of incision.

the subcutaneous tissue, the anterior rectus sheath, and the rectus muscle are divided in the line of the incision as far out as the outer border of the rectus abdominis (Fig. 2). The medial two-thirds of the posterior rectus sheath with the underlying peritoneum is cut in the same line, then the transversus abdominis is split laterally in the direction of its fibres.

When cutting the fibres of the rectus muscle, branches of the superior epigastric require ligation one-third and two- 
thirds of the way across the muscle. More length is seldom required laterally unless it is desired for mobilizing the duodenum. It can be obtained by splitting the external and internal oblique and the transversalis muscles in the direction of their fibres. This incision over the gall-bladder gives an excellent exposure of the biliary passages and it is

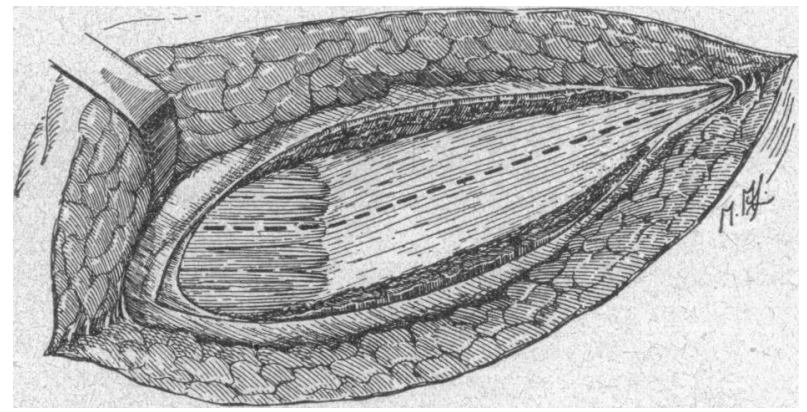

FIG. 2.-The incision completed.

easy to explore the common bile duct and, in the average case, to pass the standard Desjardins forceps down this duct. For a barrel-chested patient with a high liver I have a more curved pair of Desjardins forceps available. Should more room be required medially the incision is extended through the linea alba into the left rectus sheath. As the incision lies between the eighth and ninth intercostal nerves, these nerves are in no danger of injury and do not have to be identified (Fig. 1). Should the lateral margin of the incision be made a little low the ninth intercostal nerve is seen lying on the posterior layer of the rectus sheath near its lateral margin. The direction of the ninth nerve at this point is that taken by the ninth rib in the axilla (Fig. 1).

\section{Anatomical Points}

Identification of Ninth and Tenth Costal Cartilages

The tenth costal cartilage can be felt by running the finger outwards along the smoothly curving lower costal margin till a definite step is felt, which is caused by the tenth costal cartilage. Fig. 1 shows the usual way the tenth cartilage turns up to form this step. In two cases the tenth rib was floating and did not articulate with the ninth, reminiscent of the three floating ribs of the anthropoids. The narrow spear-like tip of the ninth costal cartilage can be felt only by taking the ninth costal cartilage between the right finger and thumb, where it is lying along the postero-inferior border of the costal margin, and, by moving it, to feel with the other hand its sharp tip where it articulates with the broad eighth costal cartilage. This identification of the tip of the ninth is not a necessary step of the operation, but it is of interest in the diagnosis of gall-bladder tenderness, which is situated most commonly 1 in. $(2.5 \mathrm{~cm}$.) below the tip of the ninth costal cartilage on a line drawn from the tip of that cartilage to the umbilicus. It is the surface marking of the infundibulum (Hartmann's pouch), where, with the patient supine, stones tend to gravitate.

\section{Direction of Fibres in Rectus Sheath}

On exposing the anterior layer of the rectus sheath, fibres of the external oblique are seen running downwards and

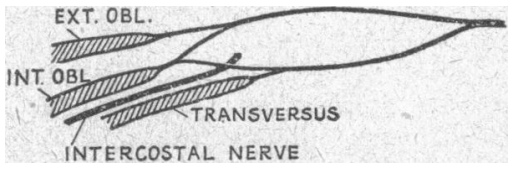

Fig. 3.- Showing fusion of the external oblique aponeurosis with the internal oblique.

the line of the incision. The external oblique aponeurosis becomes fused with the internal oblique about a quarter of the way from the lateral border of the rectus sheath (Fig. 3).
The posterior layer of the rectus sheath shows the stout fibres of the internal oblique running upwards and inwards along the line of the incision. The red muscular fibres of the transversus abdominis show through the outer third of the posterior layer of the sheath. These muscular fibres run transversely as a thin aponeurotic layer which becomes fused with the posterior rectus sheath at the junction of the outer and middle thirds of the sheath. The transversus aponeurotic fibres are by far the weaker component of the posterior rectus sheath.

Course of the Intercostal Nerves

The ninth intercostal nerve leaves the subcostal groove about the mid-axillary line and runs downwards and forwards, passing behind the tenth costal cartilage where the cartilage is turning up (Fig. 1). It lies on the transversus muscle and is below the line of the incision. The eighth intercostal nerve as it passes behind the ninth costal cartilage is directed upwards and medially and is above the line of the incision. In dividing the rectus muscle a fine twig connecting the eighth and ninth nerves can occasionally be seen. The line of incision runs between these nerves (Fig. 1).

Anatomical dissections by Davies, Gladstone, and Stibbe (1932) have shown that the rectus muscle does not receive a segmental supply from one intercostal nerve. As a result of communications in the thorax, and again between intercostal nerves when lying on the transversus abdominis muscle, any one segment of the rectus is supplied by at least three intercostal nerves. The division of any minor connecting branch in the rectus muscle does not affect the strength of the rectus muscle when the incision is healed.

I wish to thank Sir Wilfrid Le Gros Clark for drawing my attention to the work of Davies, Gladstone, and Stibbe and to the skeleton of the anthropoid, and to Miss M. C. McLarty for the drawings.

REFERENCES

Davies, F., Gladstone, R. J., and Stibbe, E. P. (1932). J. Anat. (Lond.), 66,

Kocher. Th. (1895). Operative Surgery, translated by H. J. Stiles, p. 140. London.

\section{MULTIPLE TOXIC EFFECTS OF PHENYLBUTAZONE REPORT OF A FATAL CASE}

BY

THOMAS N. FRASER, M.D., F.R.C.P., F.R.F.P.S. Physician, Rheumatism Unit, Killearn Hospital, Stirlingshire

It is almost four years since Currie (1951) first drew attention to the therapeutic effect of phenylbutazone ("butazolidin"). Since then it has been employed extensively both in hospital and in general practice, and many reports on its use, mainly in the treatment of rheumatoid arthritis, have appeared in the medical press. There is general agreement that the drug has a marked analgesic action in rheumatoid arthritis in roughly one case in three, but it is doubtful whether it has any antiinflammatory properties. As Hart (1953) pointed out, although occasionally a reduction of inflammatory swelling is seen, increase in range of movement and function tests, and decrease in stiffness, are essentially secondary to the relief of pain. Steinbrocker (1953) reported on the results he had obtained in a large series of patients on short- and long-term phenylbutazone treatment, and came to the conclusion that the drug was a powerful analgesic but that its antirheumatic effect, if any, was slight.

Since the introduction of phenylbutazone in the treatment of the rheumatic diseases numerous reports of its 